\title{
New Application of IEEE 11073 to Home Health Care
}

\author{
Isao Mizukura, Toshiyo Tamura ${ }^{*}$ Yutaka Kimura, Wenwei Yu
}

Department of Medical System Engineering, Faculty of Engineering, Chiba University, 1-33 Yayoi-cho, Inage-ku, Chiba 263-8522, Japan

\begin{abstract}
We propose a new home health care network for the acquisition and transmission of data from ordinary home health care appliances based on IEEE11073. In this study, we develop a standard protocol for data collection and a simple interface to accommodate different monitoring systems that make use of different data protocols. The system provides for one-way data transmission, thus saving power and conforming to Japanese pharmaceutical law. Our standardized protocol was verified during a 1-year field test involving 20 households in Japan. Data transmission errors between home health care devices and the home gateway were 4.21 per a day with our newly developed standard protocol. Over a 1 year period, we collected and analyzed data from 241,000 separate sources associated with healthy, home-based patients and chronically ill, clinic-based patients, the latter through physician intervention. We evaluate some of the possible applications for collecting daily health care data and introduce some of our findings relating primarily to body weight and blood pressure monitoring for elderly subjects in their own homes.
\end{abstract}

\section{INTRODUCTION}

The increase in the elderly population and the prevention of lifestyle diseases such as hypertension, dyslipidemia and diabetes highlight the importance of home health care. The aim of home health care is to make patient care more readily available and to reduce hospital admissions. Generally, a home health care system consists of sensor interfaces, a data collection system, and a data network to evaluate the health condition of individuals and transmit it to either hospital or care facilities. The sensor devices used in home health care must be simple, safe, inexpensive, and unobtrusive. Many recently developed home health care devices have most of these characteristics but also have drawbacks in that they may disturb normal living patterns or may not be sufficiently effective in their monitoring functions. Installed and wearable monitoring systems can collect health data in the home, facilitate the prediction and prevention of disease, and perform early diagnosis $[1,2]$.

The characteristics of the devices used for data collection in home health care depend on their purpose and the environment in which they are employed. Monitoring of vital signs such as heart rate, blood pressure, and respiration is required for elderly patients and for those with chronic and terminal diseases. The devices must be fairly simple, and the patients must be unaware that data collection is taking place. Once patients are discharged from hospital, they may be monitored to capture parameters similar to those measured in the hospital. The devices used in the home and in the hospital do not differ significantly. With simple training, blood pressure monitoring in the home can be as precise as that in the hospital.

*Address correspondence to this author at the Department of Medical System Engineering, Faculty of Engineering, Chiba University, 1-33 Yayoicho, Inage-ku, Chiba 263-8522, Japan; Tel: +81432903050; Fax: +81432903050;

E-mails: tamurat@faculty.chiba-u.jp, E-mail: tamurat@ spice.ocn.ne.jp
A system for health monitoring in the home has been considered for health management and disease prevention. The onset of lifestyle diseases such as hypertension, dyslipidemia, and diabetes is highly correlated to daily activities such as physical exercise, sleep, and nutrition. Daily monitoring is important for preventing such diseases and for achieving a healthier quality of life. Although the monitoring of daily activities is not well established in evidenced-based healthcare, there have been many attempts at installing sensors and transducers to monitor daily home life [3].

Data transmission is another important issue because each home device uses a different protocol. We must consider simple plug-and-play devices so that clients can connect home health care devices without difficulty.

In this paper we propose standardized application-layer protocols based on IEEE 11073 for communication between home health care measurement devices and a home gateway, and we evaluate the performance of these protocols with a field test. We examine some possible uses of daily home health care data and present major findings based on an analysis of our data.

\section{PROPOSED SYSTEM}

\subsection{System Configuration}

The proposed home health care system is divided into four parts as shown in Fig. (1). The first part is the sensing system. The second part is the data acquisition system located between the health care monitoring devices (HMD), used for monitoring physiological parameters, and the home health care coordinator device (HCD) used for data collection. The third part of the system is the data transmission system that links the HCD with the central server and the data storage backbone system via the Internet. The fourth part is the web system, which connects the web server to the subject's personal computer and the terminals used for home health care interventions by physicians, 

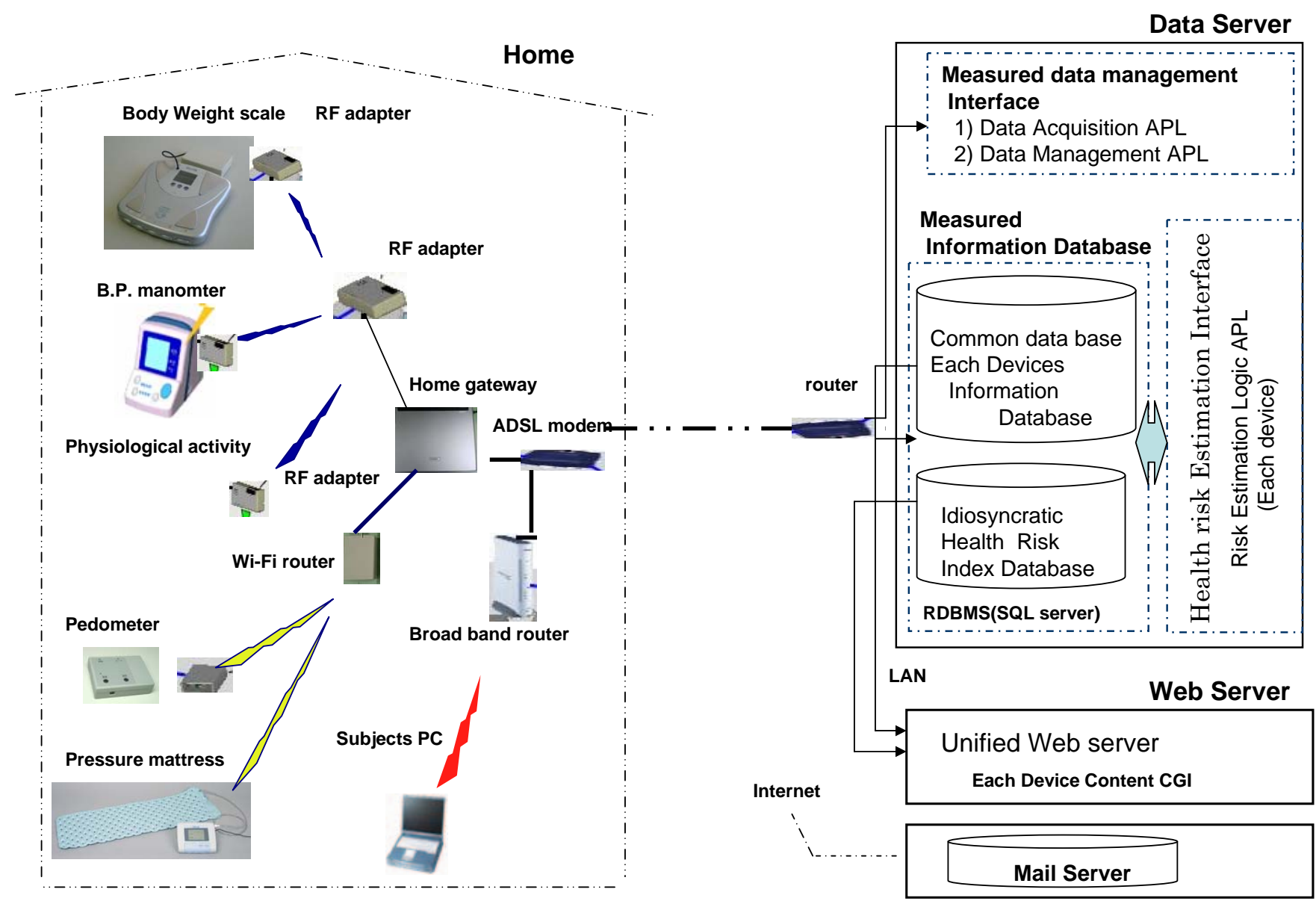

Fig. (1). System configuration.

administrative dieticians, and physical trainers whose access has been authorized by the subjects.

\subsubsection{Data Acquisition System with Home Health Care Devices}

Devices and parameters were selected especially for monitoring the progress of lifestyle-related diseases. Specifically, the body weight was used to judge obesity; blood pressure was used to assess hypertension; urine sugar was used to assess diabetes [4-6]; and a step counter was used to measure physical activity.

\subsubsection{Data Communication Protocol}

As described above, the physiological data collected from different devices have different formats and protocols. This indicates the need for a new universal communication protocol. A plug-and-play device permits multiple connections, is easy to use, and provides device compatibility among home health care devices. The client must purchase different communication adaptors because each commercial home health care device currently has its own communication system. We need to standardize the communication system for multiple monitoring.

In addition, it is difficult to combine or analyze the data from different instruments because each device has its own data format. For example, plotting a simultaneous graph of body weight and blood pressure is problematic because the protocols, formats, and time stamps produced by the body weight scale and blood pressure manometer are different. Overcoming this problem requires physical connectivity and application layer interoperability. Application layer interoperability allows diverse devices to synchronize their operational behaviour and operating states, and to intercommunicate clearly using common message syntax, data types, encoding rules, and nomenclature. Most systems are based on the Open Systems Interconnection (OSI) sevenlayer model.

As previously mentioned, the home health care system should be easy to use and capable of monitoring regular or irregular conditions, intermittent or continuous data, and small or large data sizes under a range of circumstances.

Multiple monitoring requires the transmission of much more data and increases the probability of collision. As shown in Fig. (2), the required bandwidth is much higher than when only limited data, such as blood pressure readings, are transmitted. Common unsynchronized data transmission systems such as CCITT V23 or ARIB STD T67 are not acceptable for a home health network because of the increase in data volume and collision rate.

The network connecting multiple HMDs (end devices or clients) to a single HCD (coordinator or server) has a simple star topology. Radio-frequency circuits in the HMDs (blood pressure monitor, body weight scale, and physiological data 


\section{Timescale}

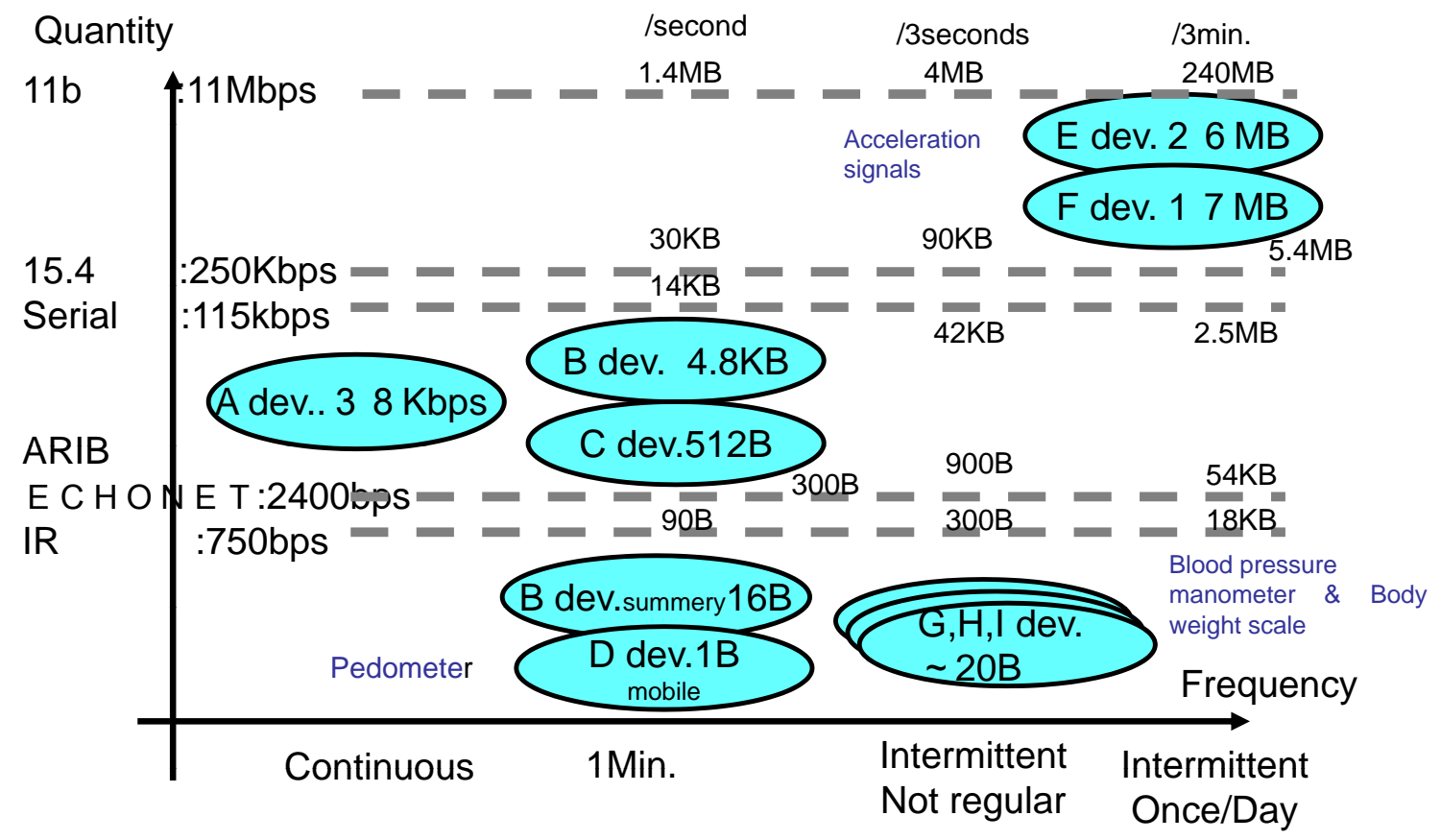

Fig. (2). Summary of physiological parameters and data transmission time.

sensing unit) sleep when they have no data to send. These devices are capable only of data transmission and are not designed for full-function communication protocols. Note that unlike traditional ad hoc wireless networks, the HMD in this topology is a master and the HCD is a slave. Masterslave pairs have the opposite function than they do in a conventional system. The MHD master asks to send data to the HCD slave. Thus, whereas the masters do not always need to be powered up, the HCD must always be on. In addition, the HCD has a client-server relationship with the central server. The HMD transmits the data that were measured by sensor interfaces to the HCD, and the HCD then retransmits that data to the central server.

The system is shown conceptually in Fig. (3). We restricted our attention to the application protocol (i.e., the data file format and the communication procedure) in the OSI seven-layer model. The transport layer and the layer below are based on wireless technology that is currently developing rapidly and increasing in speed [7,8]. We chose the Zigbee $[9,10]$ wireless communication standard, mainly because of its low power consumption. The Zigbee stack tasks are defined in the lower layers of the ISO/OSI model. Detailed information on how the application layer works in cooperation with the HMD and HCD is given in the following subsection.

\section{A) Basic Requirements of the Application Layer Protocols}

In our system, we combined the application, presentation, and session layers into a health care application protocol (HAP) and serial layer. We selected element names by considering the nature of the health care monitoring involved.

Disease-related metabolic syndrome is a general term that includes several diseases: hypertension, diabetes, dyslipidemia, and obesity. Several health care parameters are measured to evaluate the progression of these diseases. Each parameter has its own measurement timing, frequency, duration, and volume. In addition, some parameters depend on others. For example, the impedance of a weak current in the body can be used calculate body fat when combined with the age, height, and sex of the subject. The data acquisition system must be able to deal with a wide variety of health care parameters.

In addition, there is a wide range of HMD hardware types with different levels of CPU capability, battery capacity, device lifetime, and portability. The system must be able to communicate with such devices for data acquisition. Thus, the application protocols include device descriptor items and lightweight software for equipment with poor $\mathrm{CPU}$ capability and battery life.

In general, each HMD and HCD has its own communication methods, and intercommunication depends on the communication distance, protocol, and hardware. Both the HMD and the HCD must be able to adapt to the rapid advance of technology in the lower levels of the OSI model, especially for short-distance radio communication.

In addition to diversity and extendibility, the basic requirements of the application layer protocol for HMDHCD communications require careful consideration of the following:

1) The data measured by the HMD must be transmitted accurately. The main role of the HAP is to reduce the communication error rate between the HMD and HCD.

2) Newly developed HMDs measuring new monitoring parameters must work with existing HCDs. 
HMD

\begin{tabular}{|l|l|}
\hline application & Upper layer application \\
\cline { 2 - 3 } presentation & Protocol module \\
\hline session & $\begin{array}{l}\text { Lower layer wrapper } \\
\text { software }\end{array}$ \\
\hline transport & IEEE 802.1 HLLI \\
\hline network & IEEE802.2 Logical link \\
\hline data-link & $\begin{array}{l}\text { IEEE802.11 } \\
\text { CSMA/CA }\end{array}$ \\
\hline physical & IEEE802.11b PHY \\
\hline OSI model & \multicolumn{1}{|l|}{ IEEE 802 } \\
\hline
\end{tabular}

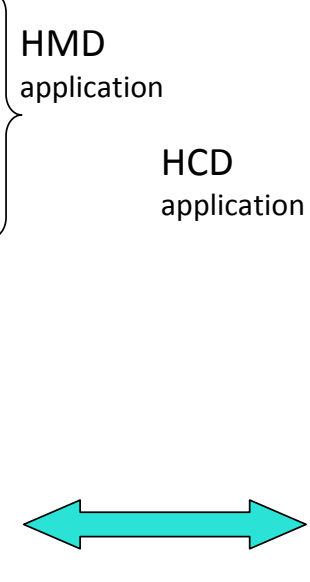

HMD

application

Fig. (3). Vertical description of the layered device architecture relative to OSI model.

The HMD and the HCD must be able to query each other to obtain health care information.

4) Lightweight communication software is required to compensate for poor CPU and battery capacity.

5) The protocol covers the top three layers of the OSI layer model.

6) Any monitoring parameter must have not only an English name, unit, and scale, but also a Japanese name.

We have developed our HAP based on the above considerations.

\section{B) Reduction of Data Length and Communication Error Rate}

Generally, health care data contain a parameter name, unit, and scale information in every data transaction. To reduce the amount of data transmitted, we developed a new a key value pair method based on Extensible Markup Language without prior definition. Using the concept of keys and values without prior definition, it was possible to reduce both the data length and the communication error rate.

The application layer protocols we developed for HMDHCD communication reduce the total amount of data transmitted, enable new devices to join the system, and permit the exchange of new HMD definitions. This is because of three technologies. First, each HMD holds its own byte name $(\mathrm{BN})$ allocation table containing the $\mathrm{BN}$ code and measuring parameters (name, unit, and unit scale).

Using the example of the body weight scale, the device can transmit the information with only one byte after the registration; by prior definition, byte 0x0c represents (name $=$ bodyweight, unit $=\mathrm{kg}$, scale $=0.1$ ). We could reduce data transmission quantities from 13 to 1 byte by prior registration. BNs can be assigned to not only the name, but also to the unit and scale.

Bodyweight (10 byte, ASCII code $)+\mathrm{kg}$ ( 2 byte, ASCII code $)+0.1$ ( 1 byte, binary $)=13$ bytes $\rightarrow 0 x 0$ c ( 1 byte, binary) $=1$ byte name
Second, the HMD sends the BN allocation table to the HCD (coordinator) using the named device information registered in the communication service before data transmission. Finally, after the BN allocation table has been recorded in the HCD, the HCD is ready to accept data from the HMD.

This protocol requires the definition of reserved words because it is designed to work with many kinds of HMDs. Through the definition of reserved words, systems can preserve logical consistency when one type of HMD replaces another. The scope of the definitions encompasses BN, personal health care attributes (e.g., the age and sex of clients), device operating information, and the device's remaining battery life.

The definition of reserved words follows ISO/IEEE 11073-10101, Health informatics-point-of-care medical device communication-Part10101: Nomenclature.

\section{C) Functional Design of the Application Layer}

We have defined four communication procedure patterns in HAP. The application layer service includes device registration, information registration, data communication, and information inquiry services.

Device Registration. This is used to register a new HMD in the system. The registered HCD information used by this function includes communication software version ID, manufacturer's name, product ID, serial number, individual $\mathrm{BN}$, and device name. Each data element has a code discrimination identifier. An HMD can easily join the system after executing this service. The function is made up of two parts: the device registration notice from the HMD to the HCD and the device registration request from the HCD to the HMD.

Device Information Registration. This includes the protocols required to achieve data compression by the prescribed $\mathrm{BN}$ method and the required $\mathrm{BN}$ allocation table. The device's information registration service is the service to create that table. The table consists of a number of health care parameters, each of which has its own BN code. BN codes have the following attributes: English name, Japanese 
name, unit, scale, and accuracy. Each attribute is described by a data length (DL, 2 bytes), data discriminator (DD), code discriminator (CD), and data value (DV).

Each HMD sets its own BN randomly; there are no reserved $\mathrm{BNs}$. The required items in the $\mathrm{BN}$ allocation table include personal ID (identifier of the person in the home); measurement date; and the height, age, and gender of the person. These items make up the system common name.

Data Communication Service. The HMD data format consists of a number of data fields. A data field contains the $\mathrm{DL}$, the $\mathrm{BN}$, and the $\mathrm{DV}$. The data fields are generally assigned to personal ID, measurement time, $\mathrm{BN}$, and data value. The first data field is assigned to the personal ID.

Information Inquiry Service. The HMD must know the current time so the data acquired by the health care system can be analysed. The HMD can obtain this from the HCD using the information inquiry service. The second function of this service is to replace the HMD. The HMD can obtain the items required from the HCD to set its common name in the system using the information inquiry service. The protocols carry the master table of system common names. The third use of this service is to obtain data measured by other devices. The HMD can obtain the measured data to describe the request about the unique device name (UDN) and selected BN of the other device, and transmit that information to the HCD. The protocols have three types of data communication services: indication without acknowledgement, indication with acknowledgement, and data request and response.

A data frame consists of the application layer header $(\mathrm{AH})$ and the application layer data frame (ADF). The $\mathrm{AH}$ indicates communication methods, whether divided or not, single or multiple data, service type, sequence number, and divided frame number. The ADF consists of a number of data fields. Each field has three types of formats: single data format, multiple data format, and verification format. The single data format uses mainly the data communication notice; the multiple data format uses mainly registration services; and the verification format uses mainly the verification notice and reply.

D) Serial Interface Specifications Between Application Protocols and Radio Communication Adaptor

These specifications determine the communication procedure and the serial communication framework. The communication procedure defines the retransmission algorithm and includes the retransmission interval, number of retry times, waiting-time for a response, and error handling procedure. The serial communication framework consists of three parts: serial header (SH), data length (SDL), and data contents (SDT). The SH contains information such as serial interface commands (e.g., acknowledgement and negative acknowledgement; ACK and NAK, respectively), baud rate setting, and error detection. The SDL is expressed as a two-byte binary numbers, as in the application protocols.

\section{E) Lower Layer}

We used two adaptors in the lower layers: IEEE 802.11b wireless LAN adaptors and ARIB STD-T67 energy-efficient radio-wave adapters [11]. The IEEE 802.11b wireless LAN adapters were connected to three-dimensional accelerometers, the health care mat, and the home gateway adapters. They use complementary code keying for direct sequence spread spectrum in a radio frequency (RF) range of $2.400-2.484 \mathrm{GHz}$ with standard CSMA/CA. The fundamental data transmission model is a push from the HMD (end device). The interface between the RF module and the HAP is a universal asynchronous receiver-transmitter (UART).

The ARIB STD-T67 adapters were used on the body weight scales, blood pressure manometers, physiological data sensing units and home gateway RF adapters. Their operating frequencies are $429 \mathrm{MHz}$ (channel 46) and 426 $\mathrm{MHz}$ (channel 10) using frequency shift keying at a baud rate of $2400 \mathrm{bps}$.

The interface has a sleep mode with wake-up control. The fundamental data transmission model is a push from the HMD (end device). The interface between the RF module and the devices is via a UART.

We defined a unified application framework data frame and data communication procedure in the application layer to create a common home health care interface to maintain device connectivity and interoperability. The unified application framework data frame can easily provide a unified data file format on the data server.

\section{F) Device Preparation}

We created a unique standard registration format and communication information format. Communication between the device and the gateway can take place once each manufacturer has registered the device code and the device communication code with the gateway.

The manufacturer provides the protocol embedded in the device firmware, along with wireless registration and installed programs on a CD-ROM. Several manufactures participated in this standardized protocol so that clients can use their own home health care devices.

\subsubsection{Web for Clients}

The collected data are transferred to the central server over the Internet using a secure communication protocol (secure sockets layer). The central server creates a database for each subject, and the graph of the data is displayed using the web server.

Equipment was developed by the participating companies and installed in patients' homes to monitor health conditions and serve as the gateway used to acquire the data. Subjects check their own vital signs on web pages after entering a personal ID number and password. Fig. (4) shows the main page that allows patients to access each equipment page by clicking on devices. They can monitor the condition of their own health with a graph of the data of interest for one day, one week, or one month at a time.

\subsection{Monitoring Protocol}

\subsubsection{Subjects}

For our test subjects, we chose 21 patients at the Kansai Medical University Hospital, along with 55 healthy members of their families. The mean age, height, and body weight of 


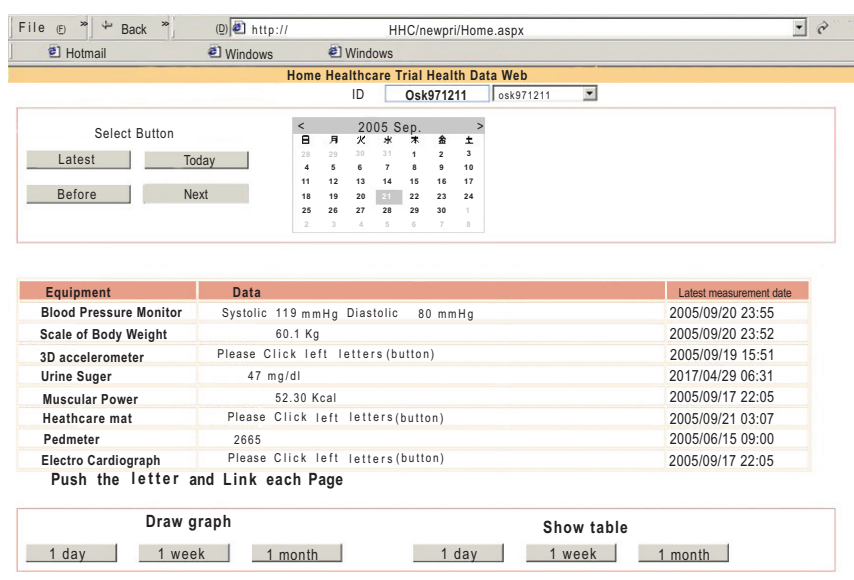

I) Check and Select Display items vB.P. FB.W. I Step Display

One Month Data View (2005/12/20 2006/01/19)

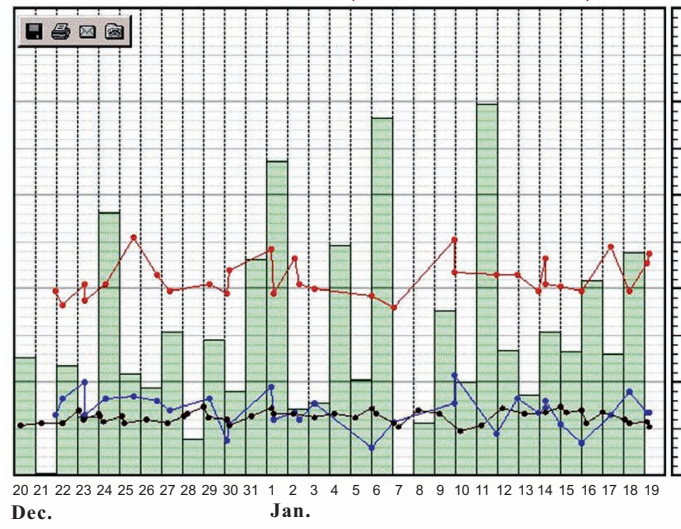

$230=73.0=25000$ $210=70.5=22500$ $190=68.0=20000$ $170=65.5=17500$ $150=63.0=15000$ $130=60.5=12500$ $110=58.0=10000$ $90=55.5=7500$ $70=53.0=5000$ $50=50.5=2500$ $30=48.0=$ P. $\longrightarrow$

Fig. (4). Individual client daily view. 
the patients were $59.1 \pm 8.8 \mathrm{yr}, 163.7 \pm 9.3 \mathrm{~cm}$, and $72.5 \pm$ $11.6 \mathrm{~kg}$, respectively. The sample included four subjects with diabetes, four with borderline diabetes, six with hypertension, four with borderline hypertension, nine with hyperlipidemia, and two with obesity. The procedure was approved the ethics committee of Kansai Medical University and we obtained written informed consent for the study from all subjects. The subjects received no direct compensation for this test although their Internet service and electricity were paid.

\subsubsection{Experimental Period}

The timing of the measurements in our field tests was carefully determined based on the daily behavioural pattern of the subjects. Intermittent measurements of vital signs such as blood pressure and body weight were made once in the morning and once at night. Urine sugar data were measured and transmitted upon demand. Data from the step counter were measured every $72 \mathrm{hr}$. The subjects sent the accumulated data every evening.

The blood pressure manometer, the body weight scale, and the urine sugar device can record data for a maximum of four people per household and each device has a personal identifier button for the client to push before using the device. The personal IDs on each device must thus be matched with the ID of each individual family member. All devices except the scale automatically transmit the data after measurement. The scale has a button that the subject must press before the data are sent to the HCD. Our operational system test with 20 families lasted four weeks after an explanation of the monitoring protocols and two months of system debugging.

\subsubsection{Protocol Evaluation}

There are many variables in a wireless communication system including baud rate, carrier frequency, modulationdemodulation method, redundancy method, error correction method, data encryption-decryption method, power control, and countermeasures for reflections and radio propagation. However, application protocols can deal with a restricted set of considerations such as data transmission certainty, data efficiency, cost efficiency, and technical feasibility. The most important of these is data transmission certainty because HAP accepts one-way communication procedures such as data indication without acknowledgement.

We used two error detection methods: the subject's daily check and the intervention group check. For the former, each subject checked his or her data once a day. In the latter, members of the intervention group at the Kansai Medical School checked the data of all subjects. When no measurements were received from a subject for more than three days, the intervention group would ring the subject and ask why.

A communication error was deemed to have occurred when the wireless communication adapters failed to operate with repeated retries or when data content errors occurred. When this happened, the system automatically sent an error message to the system manager by email. The system manager then diagnosed the cause of the error and classified it as a device error, a problem between the HMD and the

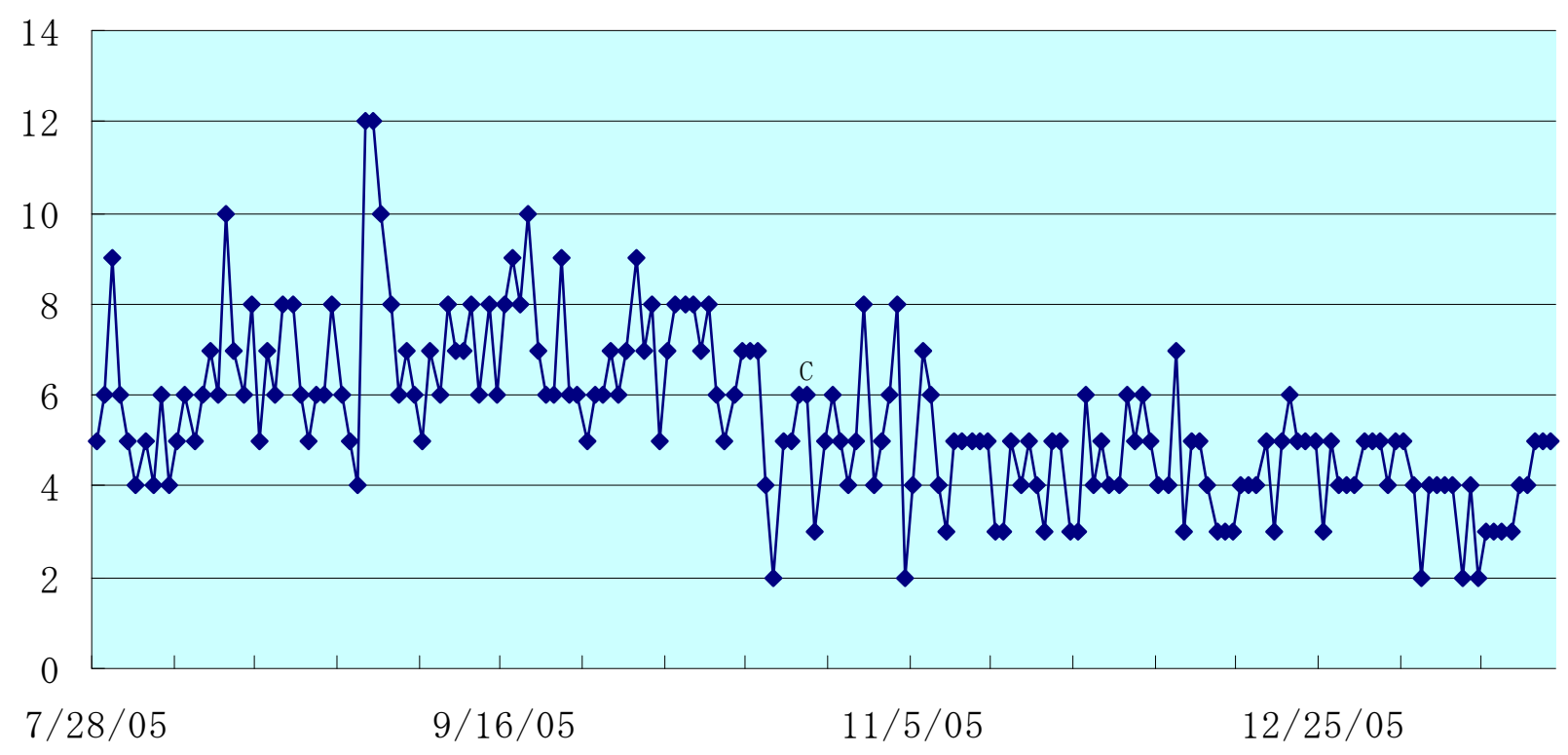

\section{Date}

Fig. (5). Transmission error rate in the second protocol version. 
HCD, or a problem between the HCD and the relay host.

We designed two protocols that differed in the communication services and communication procedure patterns provided. The first version of the protocol included only one communication service (the data communication service) and only one communication procedure pattern (data indication without acknowledgement with $\mathrm{BN}$ methods). The second version of protocol added two communication procedure patterns: indication with ACK and a subset of request and response (only latest data request).

The communication interfaces of the body weight scale, blood pressure manometer, and physiological data sensing units were not changed during the field test. These devices used the energy conservation radio-wave adapter ARIB STD-T67. Only the interfaces of the three-dimensional accelerometers and health care mats used our application protocols with IEEE802.11b wireless LAN adapters.

We compared six months of stable data for each protocol version and compared the performance of the two protocol versions using a two-sample $t$-test with Welch's correction.

\section{RESULTS}

\subsection{Data Acquisition}

In the 20 families that participated in the field test, 76 subjects registered their ID numbers; this included 21 borderline-type subjects. After four weeks of operational system testing, 48 subjects agreed to join the field test and signed the consent form; this again included 21 borderlinetype subjects. Of the 48 subjects, 39 had been weighed 100 times throughout the year; this included 20 of the borderlinetype subjects. The participation rate was greater than $81 \%$ and the participation rate of the borderline-type represented more than $95 \%$ of those who agreed to participate. However, 28 of the initial 76 subjects could not participate in the field tests that involved measurements at regular intervals, so their measurements were taken at arbitrary times. The level of involvement in the field test was greater than $55 \%$. Out of the 28 participants who were measured at arbitrary times, 19 were younger than 30 years old.

In our test population, 19 healthy subjects continued the measurements, primarily of body weight and blood pressure. The borderline-type subjects had a greater tendency to participate in measurements with various kinds of devices.

We obtained 91,714 automatic measurement data samples in about one year of field testing without any reward. The number of samples per family obtained during the year by the body weight scale, blood pressure manometer, physical activity monitor, step counter, and pressure mattress are shown in Table $\mathbf{1}$.

\subsection{Error Rate}

The reasons for transmission errors were radio wave interference; the electromagnetic environment; reflections of radio waves from the floor, wall and stairs; and the power of the radio wave communication driver.

Fig. (5) shows the error rate for communications between the HCD and the HMD.

Compared to the first version of the protocol, the second version reduced the number of communication errors by about $40 \%$, from 7.06 to 4.21 times/day. The $t$-value of the two-sample $t$-test with Welch's correction was 11.43 and the calculated DOF was 106.64. This indicates a significant difference between the two protocols.

Table 1. Number of Measurements in the Experimental Stage

\begin{tabular}{|l|c|c|}
\hline \multicolumn{1}{|c|}{ Access Time } & $\begin{array}{c}\text { Total Number of } \\
\text { Measurements }\end{array}$ & $\begin{array}{c}\text { Number of } \\
\text { Measurements } \\
\text { Per Family }\end{array}$ \\
\hline \hline Body weight scale & 15,940 & 797 \\
\hline Blood pressure manometer & 15,225 & 762 \\
\hline Physiological activity monitor & 17,679 & 884 \\
\hline Pedometer hours & 24,620 & 1,231 \\
\hline Pressure mattress hours & 18,250 & 931 \\
\hline Total & $\mathbf{9 1 , 7 1 4}$ & $\mathbf{4 , 5 8 5}$ \\
\hline
\end{tabular}

The reference communication error rate using the ARIB STD-T67 energy conservation radio-wave adapter during first protocol and second protocol was 1.66 and 1.53 times/a day. The $t$-value of two-sample $t$-test with Welch's correction was 0.578 and the DOF was 12.76. This means that the error rate of the reference communication was no different in the two periods at a $20 \%$ significance level.

The two results above show that the return acknowledgement procedure and the subset of request and response procedure in the application layer improved the data transmission certainty with retry control, redundancy control, and error check in the lower layer levels.

\subsection{Web Interface Example}

Fig. (6) shows a typical example of a blood pressure record. In the first six months, the subject monitored the blood pressure in the evening before going to bed. After intervention by a physician, the subject measured the blood pressure twice a day, once in the mooring after waking up and once in the evening. The difference between the morning and evening blood pressure caused ischemia. Blood pressure measurements over time may indicate physical problems and these data are needed to predict and prevent the disease.

\section{DISCUSSION}

We proposed a new application-layer HAP to reduce the data transmission error rate for communications between the HMD and the HCD. The primary means of reducing errors included a reduction in the overall volume of data transmitted and a data communication procedure with an acknowledgement in the application layer. The HAP makes it easy to add HMDs to systems through device registration and device information on CD-ROM. This means that the system can continue to measure parameters using other vendors' devices if the manufacturer of a current device stops production. The protocols maintain the interconnectivity of end devices.

A reduction of data transfer length is essential for reducing the error rate in radio wave communication. It can reduce data interference and data transfer time. Returning the ACK or NAK during the communication procedure in the 


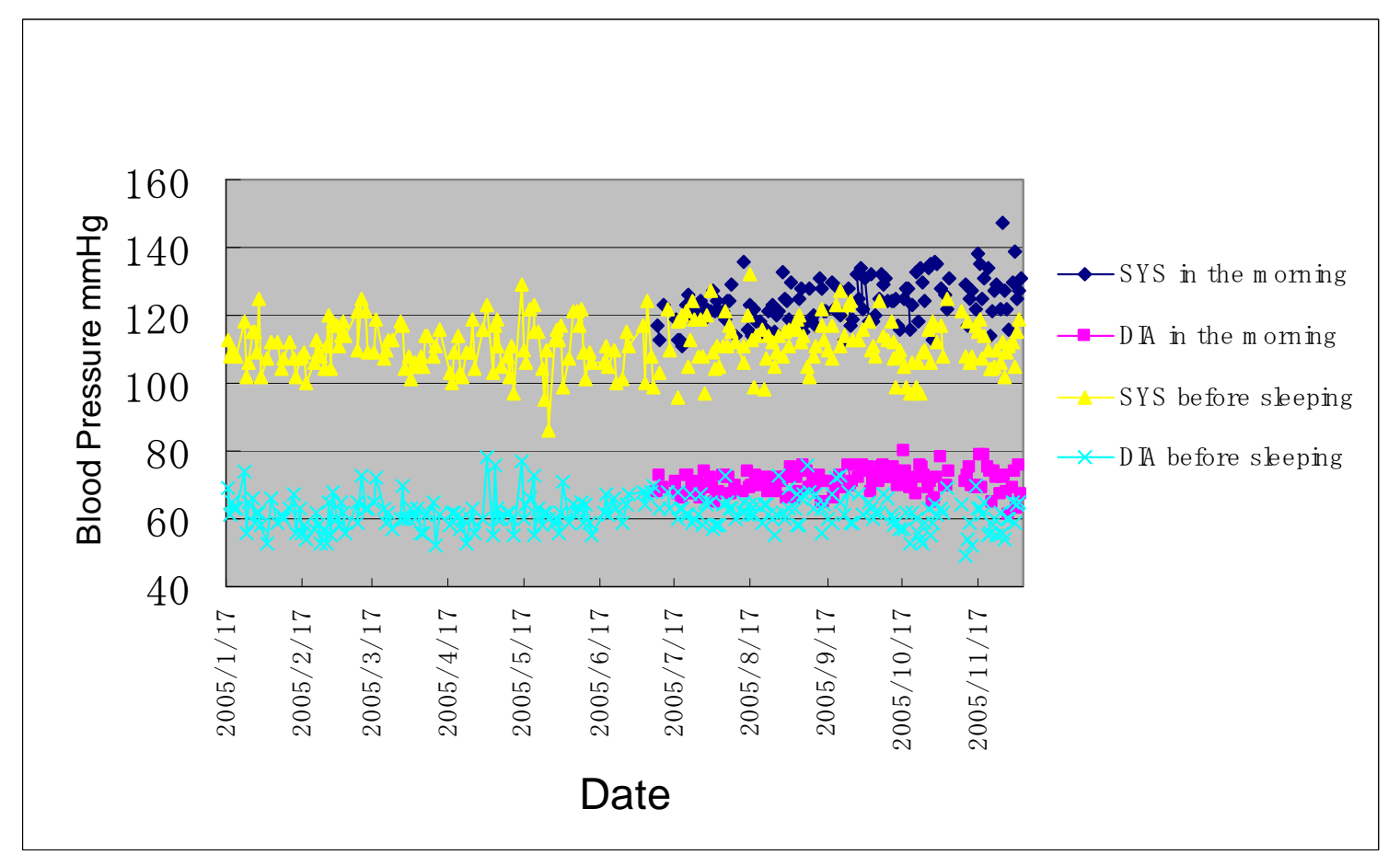

Fig. (6). Blood pressure as a function of time.

application layer is important to reduce the total amount of data transmitted. Many medical remote sensors in Japan use one-way communication with triple data redundancy from the HMD to the HCD, and HCD chooses the correct data using the two-out-of-three rule. Because the first protocol version adhered to this standard, the transmission time and total transmission quantity increased, raising, in turn, the risk of data interference and communication errors. The second protocol version returned ACK or NAK and used inquiry and response procedures to eliminate the need for redundancy. This decreased the error rate $40 \%$ from 7.06 to 4.21 times/day. Our implementation of standardized application protocols between the HMD and HCD was successful. These protocols maintained the adaptability and flexibility of incorporating new parameters and new devices into the systems. The protocols are not computationally intensive and can deal with many types of equipment with diverse parameters.

The $868 / 915-\mathrm{MHz}$ channel is not authorized for home healthcare applications in Japan, and the power of $2.4-\mathrm{GHz}$ radios is restricted to $10 \mathrm{~mW} / \mathrm{MHz}$ by the Ministry of Internal Affairs and Communications (MIC). To achieve good radio propagation in patient homes, it is necessary to convince the MIC to assign an appropriate band for home health care applications so that the cost of the devices can be reduced. Another solution to this problem could be to have the HMD (end device) and HCD (coordinator) support a cluster tree or mesh topology [12]. These methods would work with multi-hop communications and routing control at the expense of redundant messages, large time delay, and power expenditure. This last factor is significant because the scale and pedometer are both battery powered. In addition, the number of HMDs in a house would not be large enough for multi-hop communications.
Other solutions to this problem include using highsensitivity diversity antennas and transmitter power control in the communications interfaces [13]. In any case, the interfaces in the lower layer are a serious problem. Once RF modules based on IEEE 802.15.4 [14,15] become commercially available, the use of a cluster tree topology network will make construction of communication systems easy. The essential solutions to this problem involve transmitter power and frequency.

The continuous measurement of a person's health condition is now possible at home without the intervention of medical experts. However, more data are still required to demonstrate that this is practical. The motivation to participate in measurements depends on the individual's involvement such as the event how much learn about the information of the healthcare from the physician. We are now planning a new system increase motivation using information technology.

\section{CONCLUSION}

We have proposed a new home health care network for the acquisition and transmission of data from ordinary home health care devices. We have developed a standard protocol for data collection and a simple interface to accommodate different monitoring systems that make use of a variety of data protocols. The system provides for one-way data transmission, thus saving power and conforming to Japanese pharmaceutical law. Our standardized protocol was tested during a one-year field test involving 20 Japanese households. Data transmission errors between home health care devices and the home gateway averaged 4.21 per day with our new protocol. Over the one-year test, we collected and analyzed data from 241,000 separate sources associated with healthy people and chronically ill homebased patients, as well as clinic-based patients through physician intervention. 


\section{ACKNOWLEDGEMENTS}

This research was supported by the Japanese Ministry of Economy, Trade, and Industry and the Japanese Technology Research Association of Medical and Welfare Apparatus. Twelve companies participated in this project. We thank all the staff and the subjects. Isao Mizukura designed the project, served as assistant general manager, acted as technical editor, designed the study protocols, enrolled participants, participated in data acquisition and analysis, and drafted the manuscript. Toshiyo Tamura organized the advisory group from the Japanese Technology Research Association of Medical and Welfare Apparatus, provided us with constructive suggestions, and advised on important intellectual content for the concept of the study. Isao Mizukura had full access to all the data in this study and takes responsibility for its integrity and the accuracy of the data analysis. This work was partly presented at ITAB2007 in Tokyo.

\section{REFERENCES}

[1] American telecare, available from http://www.americantelecare. com/

[2] Telemedicine information exchange, available from http://tie. telemed.org/homehealth/

[3] Tamura T, Kawarada A, Nambu M, Tsukada A, Sasaki K, Yamakoshi K. Ehealthcare at an experimental welfare techno house in Japan. Open Med Informat J 2007; 1: 1-7.

[4] Centonze D, Guerrieri A, Palmisano F, Torsi L, Zambonin PG. Electrochemically prepared glucose biosensors: kinetic and faradaic processes involving ascorbic acid and role of the electropolymerized film in preventing electrode-fouling. Fresen J Anal Chem 1994; 349: 497-501.

[5] Anzai J, Takeshita H, Kobayashi Y, Osa T, Hoshi T. Layer-bylayer construction of enzyme multilayers on an electrode for the preparation of glucose and lactate sensors: Elimination of ascorbate interference by means of an ascorbate oxidase multilayer. Anal Chem 1998; 70: 811-7

[6] Yamanouchi T, Minoda S, Yabuuchi M, et al. Plasma 1,5-anhydroD-glucitol as new clinical marker of glycemic control in NIDDM patients. Diabetes 1989; 8: 723-9.

[7] Gutierrez A, Callaway H, Barrett R. IEEE 802.15.4 Low-Rate Wireless Personal Area Networks, Institute of Electrical \& Electronics Engineers, New York 2003.

[8] Callaway E. Wireless Sensor Networks, Auerbach Publications; New York 2003

[9] Zigbee Alliance, ZigBee Device Objects (version 1.0), 2004.

[10] Zigbee Alliance, ZigBee Device Profile (version 1.0), 2004.

[11] Association of radio industries and businesses, Overview of standards and Technical reports available at http://www.arib.or.jp/ english/html/overview/index-imp.html.accee on 20 March 2009

[12] Zigbee Alliance, Network Specifications (version 1.0), 2004.

[13] Rosberg Z. Optimal transmitter power control in interleave division multiple access (IDMA) spread spectrum uplink channels. IEEE Trans Wireless Commun 2007; 16: 192-201.

[14] LAN/MAN Standards committee of the IEEE Computer Society, 802.15.4 Part 15.4: Wireless medium access control (MAC) and physical layer (PHY) specifications for low-rate wireless personal area networks (LR-WPANs), IEEE New Yorkspecificaiotn ver., 2003.

[15] Heile B. IEEE 80215 Working group for WPAN, available at http://www.ieee802.org/15. access on 1 March 2009 